\title{
Monitoring the Vela Pulsar with a Phased Array Feed (PAF) Receiver
}

\author{
John M. Sarkissian, John E. Reynolds, George Hobbs \\ and Lisa Harvey-Smith
}

CSIRO Astronomy and Space Science, Australia Telescope National Facility, PO Box 276, Parkes NSW 2870, Australia

\begin{abstract}
We have observed the Vela pulsar for 1 year using a Phased Array Feed (PAF) receiver on the 12-m antenna of the Parkes Test-Bed Facility (PTF). These observations have allowed us to investigate the stability of the PAF beam weights over time, to demonstrate that pulsars can be timed over long periods using PAF technology and to detect and study the most recent glitch event that occurred on 12 December 2016. The beam weights are shown to be stable to $1 \%$ on time scales on the order of three weeks.
\end{abstract}

Keywords. pulsars: general, instrumentation: miscellaneous

\section{Introduction}

The Parkes Test-Bed Facility (PTF) was commissioned in 2009 as a test platform for the CSIRO's program of developing PAF receiver technology. We have conducted regular monitoring of the Vela pulsar and report here on our observations and on the stability of the PAF system. Our data set provides the first long-term, timing observations of a pulsar using a PAF.

The PTF comprises a 12-metre fully-steerable antenna made by Patriot Antenna Systems, with the first of the ASKAP Mark-I PAF receivers mounted at prime focus $(\mathrm{f} / \mathrm{D}=0.4)$. The PAF is of the chequerboard design (Hay \& O'Sullivan 1993) and has 188 individual ports which sample the electric field in the focal plane in two orthogonal linear polarizations. The analogue signals are digitized and combined in digital beam-formers to form up to nine dual-polarization individually-steerable beams. We make use of builtin diagnostic tools that allow limited capture of beam-formed voltage (base-band) time series. Sixteen contiguous frequency channels, each of $1 \mathrm{MHz}$ bandwidth, can be captured continuously in 1-second bursts, with a dead-time of several seconds for read-out, the length of which depends on the number of captured beams.

\section{Vela Observations}

Observations of the Vela pulsar (PSR J0835-4510) were performed daily, depending on the availability of the PTF, for one full year. Each observation typically lasted 20 minutes of which $0.88 \mathrm{~s}$ of every $6 \mathrm{~s}$ would contain actual data. Immediately following an observation, the output VDIF files are converted to PSRFITS format using software specifically written for this project.

A glitch event occurred on 12 December 2016 and was quickly reported by Palfreyman (ATEL 9847) at MJD 57734.48 between 11:31 and 11:46 UTC, with $\Delta \nu / \nu=1.3 \times 10^{-6}$. Our first observation was made independently, less than 1 hour after the glitch at 12:34 UTC with our previous observation being on 11 December 2016 at 21:10 UTC. We 
Table 1. Parameters for the 12 December 2016 Vela glitch. Uncertainties are given in parenthesis and refer to the last significant digit. The top five parameters are the determined values. The bottom three parameters are derived values.

\begin{tabular}{ll}
\hline Parameter & Value \\
\hline$\phi_{g}$ & $-0.0077(5)$ \\
$\Delta \nu_{g}(\mathrm{~Hz})$ & $1.6044(2) \times 10^{-5}$ \\
$\Delta \dot{\nu_{g}}\left(\mathrm{~s}^{-2}\right)$ & $-1.21(3) \times 10^{-13}$ \\
$\nu_{d}(\mathrm{~Hz})$ & $1.29(8) \times 10^{-7}$ \\
$\tau_{d}(\mathrm{~d})$ & $0.96(17)$
\end{tabular}

Glitch epoch (MJD)

\begin{tabular}{ll}
$\Delta \nu / \nu$ & $1.43387(2) \times 10^{-6}$ \\
$\Delta \dot{\nu} / \dot{\nu}$ & $0.0077(2)$ \\
\hline
\end{tabular}

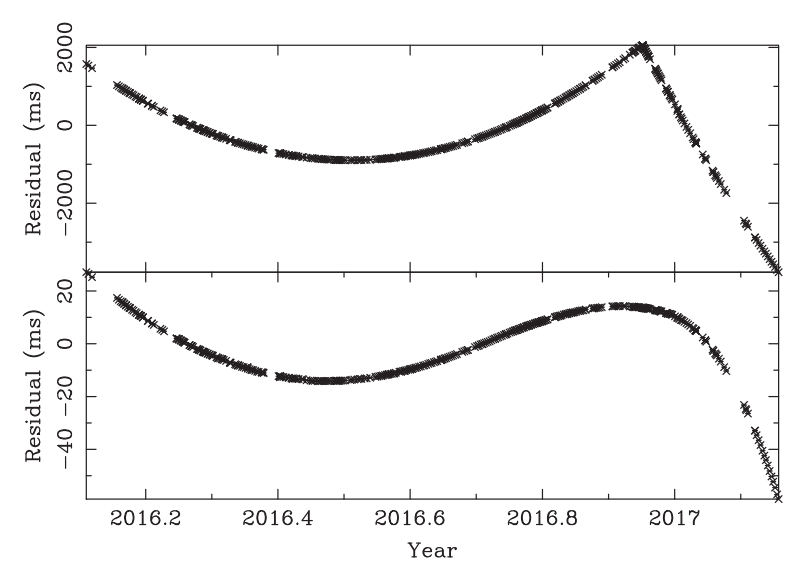

Figure 1. The full data set showing the glitch event (top); the observed timing residuals after removing a fixed frequency and time derivative only (upper panel), and after fitting for the glitch event (lower panel).

used the glitch plugin in TEMPO2 to both visualise (Figure 1) and to obtain an initial parameterisation for the glitch event. We were able to fit for the epoch of the glitch and the changes in the pulse frequency and its derivative using 20 days of observations either side of the glitch. The glitch plugin also clearly indicated a decaying term, which we modelled as an exponential recovery. See Table 1 for the resulting glitch parameters.

We have also investigated beam-weight stability by making a number of observations of the Vela pulsar with two simultaneous beams, both centred on the pulsar, with one beam formed from beam weights determined shortly before the observation, and the second from beam weights determined several days previously. We then compared the $\mathrm{S} / \mathrm{N}$ of the folded pulsar profile in the two beams. By dividing the measured $\mathrm{S} / \mathrm{N}$ of the older beam by that of the newer beam, any ageing effects should appear as a decline in this quotient over time. From our analysis, it was clear that the beam weights were stable to $1 \%$ over a period of order three weeks.

\section{Acknowledgments}

The Parkes Test-Bed Facility is part of the Australia Telescope which is funded by the Commonwealth of Australia for operation as a National Facility managed by CSIRO. 